\title{
H.-N. Macha zum 60. Geburtstag
}

Am 3. November 1940 wurde H.-N. Macha in Quedlinburg geboren. Der Vater fiel 1941 im Krieg. 1950 zog die Familie nach Bad Salzuflen. Abitur am humanistischen Gymnasium in Herford 1960 und Beginn des Studiums in Berlin. Anfangs studierte er Germanistik und Philosophie, dann definitiv Medizin in Berlin und Heidelberg. Staatsexamen und Medizinalassistentenzeit in Berlin. Promotion bei Prof. Rolf Schröder über „Einfluss von Diphenylhydantoin auf die Hämodynamik beim akuten Herzinfarkt“.

1968-1971 arbeitete er als Assistent im Institut für Pathologie im Klinikum Steglitz bei Prof. Maßhoff, einem strengen und allseits gefürchteten Lehrer. Unser Jubilar veröffentlichte seine ersten wissenschaftlichen Arbeiten über Lungenhämosiderose und hyaline Membranen beim ARDS.

1971 wechselte Dr. Macha in die kardio-pneumologische Abteilung im Klinik Steglitz. Prof. R. Schröder und Prof. U. Hüttemann waren seine ersten Fachlehrer. Aus dieser Zeit stammen seine Arbeiten über IBBP-Langzeittherapie, die pharmakologischen Untersuchungen von Atropinderivaten bei COLD, die Studien über den Einfluss von Betablockern und gleichzeitiger Gabe von Betamimetika und die Untersuchungen über konditionierende Faktoren beim Asthma. Erste Publikationen von H.-N. Macha zu diesen Themen erschienen in CHEST.

Zwar erhielt er im Klinikum Steglitz eine breite internistische, kardiologische und intensivmedizinische Ausbildung, die Pneumologie kam in der Universitätsmedizin nicht vorrangig zum Zuge. Ende der siebziger Jahre gab es einen „Stau“ in der inneren Abteilung der Medizinischen Klinik. Von den 32 Stationsärzten waren 20 habilitiert, kamen aber nicht weg, und blockierten Langzeitstellen. Dr. Macha wechselte daher 1979 in das Krankenhaus Neukölln und wurde pneumologischer Oberarzt in der Kardiologie bei Prof. Wagner. Zwei Jahre später ging er als leitender Oberarzt der II. Lungenabteilung an das Krankenhaus Havelhöhe (Chefarzt Dr. Voigt). Unter Leitung dieses sehr erfahrenen und sorgfältigen Phtysiologen lernte er die Lungenheilkunde „von der Pike auf“ bis zur selbständigen Pneumologie. Schließlich gelang es ihm unter schwersten Bedingungen, im Mai 1986 als „Externer“ an der FU zu habilitieren über das Thema „Laser- und Afterloadingtherapie stenosierender Bronchialtumoren“. Sein Habil-Vater war kein Pneumologe sondern der Strahlentherapeut Prof. R. Schumacher aus dem Rudolf Virchow Krankenhaus Berlin. Mit seinen richtungweisenden Arbeiten fand er große Anerkennung bei Strahlentherapeuten wie Pneumologen. Ihm ist es zu verdanken, dass die endobronchiale Lasertherapie und die endoluminale Kleinraumbestrahlung heute unverzichtba-

Pneumologie 2000; 54: 479

(c) Georg Thieme Verlag Stuttgart · New York ISSN 0934-8387 re Bestandteile der endobronchialen Tumorbehandlung geworden sind. Er hielt im Inland wie im Ausland vielbeachtete Vorträge über interventionelle bronchologische Verfahren. Nicht zuletzt wegen seiner hervorragenden Sprachkenntnisse schätzt man ihn seither in Amerika und England als fesselnden Redner und fairen Chairman.

Im März 1986 wurde Herr Priv.-Doz. Dr. H.-N. Macha Chefarzt der II.Lungenabteilung des Krankenhauses Havelhöhe. Die Abteilung wurde Ende August 1986 im Zuge der Sparmaßnahmen des Berliner Senates geschlossen. Schweren Herzens musste er Berlin verlassen.

Im Oktober 1986 wurde H.-N. Macha schließlich Chefarzt der pneumologischen Abteilung der Lungenklinik Hemer. Zu diesem Zeitpunkt war die Klinik nicht gerade in optimaler Lage. Mehrere Millionen DM Investition musste er zunächst dem Träger abringen, um die Klinik auf Erfolgskurs zu bringen. Die Rechnung ging tatsächlich auf. Die Abteilung hat nie wieder „rote Zahlen“ geschrieben. Sie erfuhr unter der Leitung des ärztlichen Direktors H.-N. Macha einen kontinuierlichen Ausbau und Umbau zu einer vielseitigen, modernen, alle Aspekte der heutigen Pneumologie abdeckenden Institution. Die Lungenklinik Hemer mit 240 Betten verfügt heute über alle Großeinrichtungen, die man sonst allenfalls unter mehreren Dächern an Universitätskliniken findet.

H.-N. Macha fand trotz eines gigantischen Arbeitspensums in der Klinik Zeit, weitere wissenschaftliche Arbeiten zu veröffentlichen. Er initiierte und beteiligte sich an zahlreichen multizentrischen und internationalen Tumorstudien und leitete beispielsweise über einige Jahre die Sektion der Endoskopie der ERS.

Seinen Mitarbeitern hat er jede Förderung zukommen lassen. Sie schätzen und lieben ihren Chef als vorbildlichen Kliniker und Lehrer. Im Vergleich mit anderen ist er vielleicht nicht der ehrgeizigste Wissenschaftler, aber ein begeisterter klinischer Pneumologe mit einem breiten Spektrum.

Seit 1967 ist H.-N. Macha verheiratet mit der Lehrerin Anneliese Macha. Die beiden haben vier, inzwischen erwachsene Kinder (und einen Hund). Wenn der Chefarzt mal gerade nicht bronchoskopiert, reitet er auf kleinen wilden Pferden durch die Wälder von Dortmund oder Nordamerika. Er interessiert sich für die deutsche Literatur und Geschichte des 19. und 20.Jahrhunderts, die Architektur und den Denkmalschutz.

Der Unterzeichnende, der sich seit 20 Jahren als seinen Freund bezeichnen darf, wünscht ihm, im Namen aller Kollegen und aller anderen Freunde weiterhin viel Freude und Schaffenskraft.

Pal L. Bölcskei, Nürnberg 